\title{
The Future SEM Sees 3 Dimensions... Bringing Deconvolution Techniques to the Electron Microscope
}

\author{
F Boughorbel, X Zhuge, P Potocek, L de Bruin and B Lich
}

FEI, Electron Optics BV, Eindhoven, The Netherlands

The Scanning Electron Microscope (SEM) as a three dimensional imaging tool is increasingly popular for studies in neurobiology, which can be attributed to the usefulness of serial block face cutting techniques [4, 5]. Serial slicing methods based on diamond-knife cutting are, however, reaching practical limitations in terms of achievable z-resolution and voxel isotropy. This is mainly due to the difficulty of cutting sections thinner than $15 \mathrm{~nm}$ with the desired consistency. While Focused-Ion Beam serial block face imaging can improve the z-resolution to $5 \mathrm{~nm}$, this technology is restricted in terms of the total volume of material that can be processed. In this work we describe the Multi Energy Deconvolution Scanning Electron Microscope or "MEDSEM" method that achieves high isotropic resolution by a combination of backscatter image sequence acquisition and deconvolution (DC). The method was first introduced by Boughorbel et al in [1].

The method is built on improved understanding of beam-sample interaction for classically prepared resin-embedded samples. Monte Carlo simulation tools and experimental observations show that these materials exhibit high linearity allowing for the use of image formation models based on linear convolution. Furthermore the point spread function (PSF) of backscatter electrons (BSE) in these materials appears to be well confined laterally for the typically used primary energies [3]. As the range of penetration in the sample is dependent on the energy of the primary beam, acquiring an image sequence with increasing landing energies leads to the acquisition of images from increasing depth. These images contain overlapping volume information from which 3D layers can be extracted using DC algorithms (Fig. 1). The good lateral confinement of PSFs allows for restricting the DC to the $\mathrm{z}$-axis making it similar to a source separation task [2]. As the structure of the PSF is difficult to obtain experimentally it is a variable in the resulting blind DC problem. Efficient iterative methods allow for the recovery of both the depth layers and PSFs.

To verify the reconstruction results we combined this technique with classical FIB-SEM serial block imaging using the through the lens detector in BSE mode on an FEI Helios 650 DualBeam. The zresolution was controlled by switching the primary beam energy in small steps. The comparison with a high resolution FIB reconstruction showed identical structures on the studied samples proving the reliability of the $3 \mathrm{D}$ technique (Fig. 2). From this comparison the reached depth at $5 \mathrm{kV}$ was estimated to be around $200 \mathrm{~nm}$. The combination of virtual slicing using BSE DC with physical cutting offers the possibility of reconstructing very large high-resolution datasets with isotropic voxels.

The method has been developed around typical life science specimen (dehydrated, heavy metal stained and resin-embedded), prepared for serial sectioning techniques. We foresee that this technique can help in identifying areas of interest for deeper 3D reconstructions, just by collecting data from the surface. Also the method allows for the collection of high quality isotropic data, even when the specimen does not allow the physical removal of thin enough layers. 

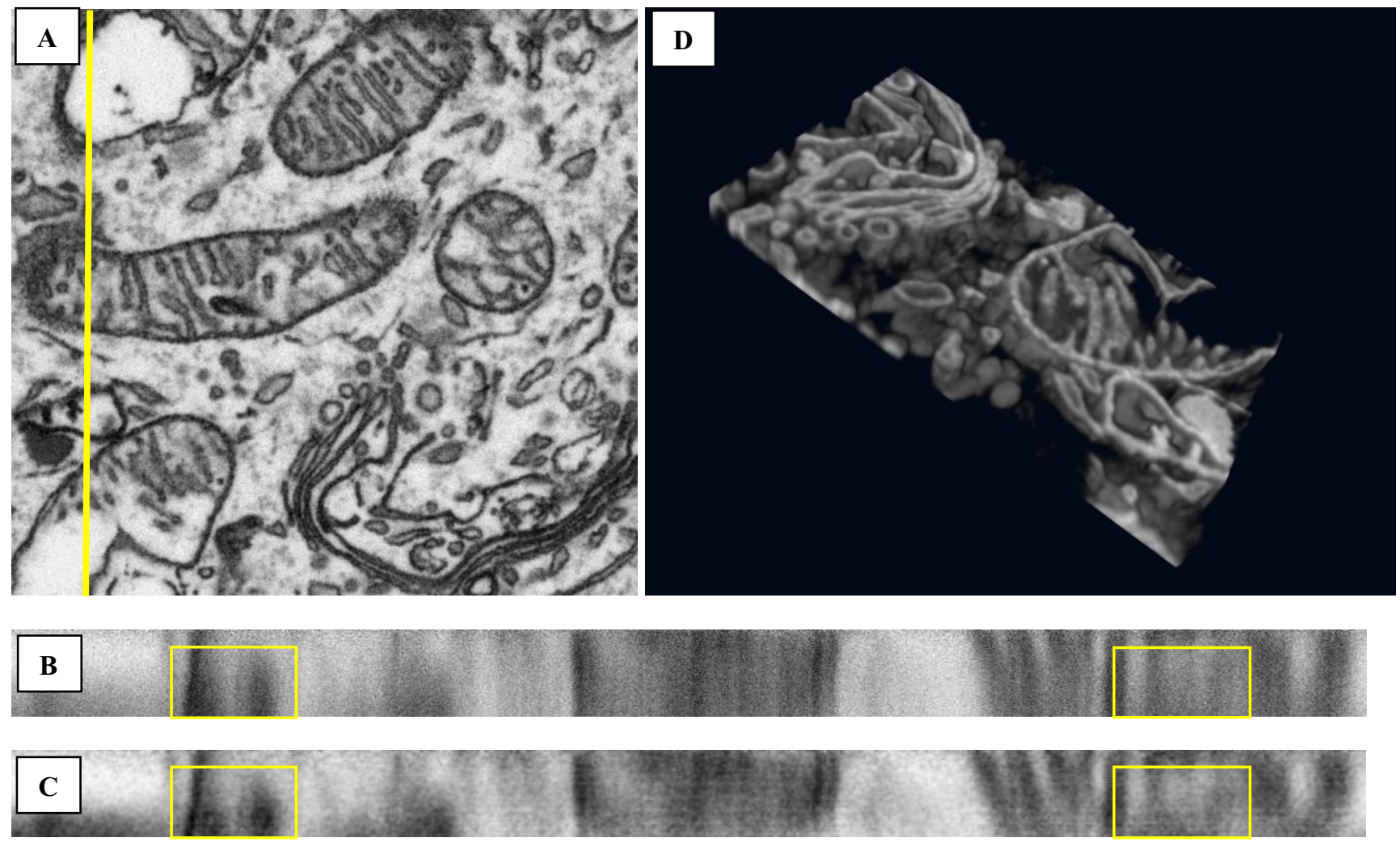

Fig 1 - Mouse brain sample multi-energy BSE image stack and reconstruction results (62 images from $1.0 \mathrm{kV}$ to $4.0 \mathrm{kV})$. The YZ Cross sections in the stack at the indicated line in the reference image (A): before deconvolution the cross section is shown in (B) and after processing in (C). New information emerges such as vesicle structure and resolved membranes as indicated by the outlined area. In (D) a three dimensional rendering of a sub-region of the reconstruction shows further details. The depth at $4 \mathrm{kV}$ is estimated to be $150 \mathrm{~nm}$.
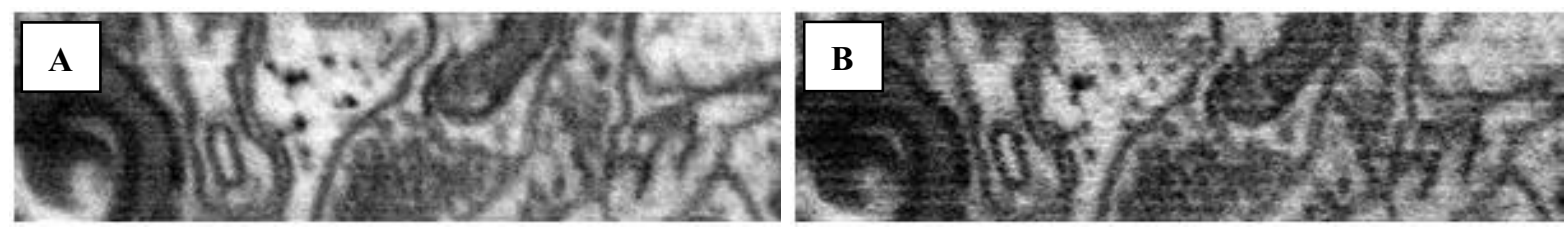

Fig. 2. Comparison of 3D reconstructions (cross sections in depth) of a $1.4 \mu \mathrm{m}$ deep region of neuropil obtained from: (A) FIB-SEM serial block face imaging ( $\sim 5 \mathrm{~nm}$ voxel), (B) a combination of removing layers of $45 \mathrm{~nm}$ with the FIB and MEDSEM computational sub-slicing using blind deconvolution.

\section{References:}

[1] F Boughorbel et al, 'SEM Imaging Method', Patent US 8,232,523 B2, 31st July 2012.

[2] P. Comon and C. Jutten, Handbook of Blind Source Separation: Independent Component Analysis and Applications, Academic Press, New York, 2010.

[3] PI Hennig et al, Journal of Applied Physics volume 102(2007), p. 123101.

[4] W Denk, H Horstmann, PLoS Biol. 2004 Nov;2(11):e329.

[5] G Knott et al, The Journal of Neuroscience, 19 March 2008, 28(12): 2959-2964.

The authors acknowledge Dr Graham Knott (EPFL) for providing the samples which were used for characterizing the MEDSEM method. 